\title{
Prospect of Applications Research on Digital Pathology Teaching Resources
}

\author{
Haiying Dong \\ Qiqihar Medical University, Qiqihar, 161006, China
}

Keywords: Teaching resources, Digital pathology, Pathology experiment

\begin{abstract}
As a new morphological teaching method, digital teaching can be treated as an important auxiliary means of pathological morphology teaching. Digital pathology teaching resources provide great convenience for medical teaching, which greatly enrich the teaching content and improve the teaching efficiency. In this paper, the main types and construction methods of digital pathology teaching resources are discussed, and the application situations, main problems and future prospects of digital pathology teaching resources are mainly studied to provide some references for the relative researchers.
\end{abstract}

\section{Introduction}

Pathology is a basic medical course to study human diseases. Its experimental teaching is an important method to link theory with practice [1]. The traditional experimental teaching mode is mainly for teachers to explain and students to listen. This disease is not conducive to students' independent thinking and hands-on practice, and the teaching effect is general. Digitalized network pathology experiment teaching is a new teaching mode which can arouse students' subjective initiative. With the rapid development of computer and network, education is also undergoing profound changes, modern high-tech has been gradually applied to morphological experiment teaching. Digitalization of images makes it possible to make traditional glass slices into digital images. We make use of modern teaching methods and establish a perfect digital teaching evaluation to improve the quality of pathology teaching. Digital experiment teaching refers to the application of computer and network technology in experimental teaching, which is a combination of modern digital system and traditional optical amplifier. It acquires high-resolution digital images by scanning and acquisition through automatic microscope or optical amplification system, and then automatically performs high-precision multi-view seamless stitching and processing on the obtained images by computer to obtain high-quality visual data for experimental teaching. The digitalized teaching of pathological experiments includes two aspects: slice digitalization and gross specimen digitalization. Digital pathology teaching resources have the advantages of non-fading, easy preservation, clear image, enlargement and reduction at will and case sharing. As a teaching method, digitization is introduced into pathology experiment teaching, which is necessary and effective supplement to some teaching slices with scarce source and few quantities. It makes up for the shortage of teaching resources and improves the teaching effect and efficiency [2].

\section{Main Categories of Digital Pathology Teaching Resources}

\subsection{Digital Gross Samples}

The study of gross specimens is helpful for students to understand the whole changes of diseases and is of great significance to understand the process of disease occurrence. The observation of gross pathological specimens is one of the intuitive links in pathological morphology teaching. With the application of computer multimedia teaching, it is very easy to obtain the image of general specimens of diseases. Many teaching units have gradually established the image database of general specimens, which greatly enriches the teaching resources of general specimens. Students can read pictures of gross specimens at any time and improve their learning efficiency. Pathological gross specimens are 
from human tissues or organs, with obvious pathological characteristics, representative, irreplaceable pathological display. It is difficult to obtain gross specimens of pathology. In order to preserve the precious gross specimens in use, image acquisition of gross specimens by digital method is also used as the basic teaching material of pathomorphological. In addition to using traditional photography technology to take photos of pathological gross specimens and digitize them, there are also 360-degree digital production of pathological gross specimens, and the establishment of network teaching platform, as well as the use of object panorama to digitize the gross pathological specimens for 3D processing, applied to pathological morphology teaching, worthy of medical treatment. It is widely used in other subjects of education. Pathology is a morphological course. To realize the network of pathological teaching, we must overcome the shortcomings of bulky and obsolete pathological gross specimen. The digitization of the gross specimen of pathology has solved this problem well. The general specimen of digitalization is vivid and vivid, and almost completely restores every detail of solid specimen. It can be used as the material of making pathology micro-lesson and mu-lesson, improve the quality and effect of micro-lesson and mu-lesson, so that pathology teaching can be better integrated into the network process [3].

\subsection{Digital Sections}

We use the full-automatic microscope scanning platform to collect images, seamlessly stitching, and generate full-field digital virtual slices [4]. Digital slices can be observed at different rates by software on a computer, and it has been proved that high-level digital slices are similar to conventional slices. Virtual section teaching, academic exchanges, doctor training, remote case consultation and so on are realized by using the network, which is not limited by time and space. According to the teaching needs, the pathology teaching and research department has established a wealth of digital slice library, which provides a large number of materials for pathological morphology teaching: digital slices with full field of vision information, clear pictures, vivid color. At the same time, it can avoid the constant loss of teaching tissue slices, and the pathological tissue can be used permanently after scanning, thus improving the utilization of teaching resources. Moreover, the use of digital slices does not depend on the microscope, but the use of the corresponding image browsing software for observation, with the mouse operation can choose any position of the slices, scaling and arbitrary magnification of magnification or reduction. Under microscope, it does not produce distortion of image information. Without microscope and glass slices, students can observe the slices from themselves, which is not limited by time and space, so as to improve learning efficiency. People from different places can discuss and express their opinions together, regardless of time and place. Digital slices are convenient for lecture teaching and collective reading. They can be displayed on the same screen or viewed on each person's monitor. The effect is the same. The group discussion is not limited by the microscope. The teacher dragged the image to demonstrate and operate in real time and interact with students and voice discussions. We use special image analysis software to analyze the whole digital pathological section or user-defined area. If the teacher uses batch mode, it has a high automatic analysis function and improves work efficiency.

\section{Construction Methods of Digital Pathology Teaching Resources}

The following steps are needed to build a teaching resource repository for pathology. To investigate the needs of teachers and students for pathology teaching resources, grasp the characteristics of pathology teaching to customize the framework of pathology teaching resources. A unified standard of resource classification is determined, and the collection and collation of relevant information is organized and implemented. Group authorization for different users. The designated person completes the classification, modification and maintenance of the data resources after the audit. A teaching resource database evaluation system was established and the resources built were evaluated. Guided by the needs of students and teachers, we can make full use of the vast space provided by computers, accessory hardware and network to access the teaching resources of different stages and objects, so as to provide students with a good learning environment, to create a good 
environment for teachers, to reduce the burden of teachers, and to improve teaching efficiency. The construction of a digital resource bank which meets the needs of pathological morphology teaching in medical colleges and universities will effectively improve the teaching efficiency. To meet the needs of current teaching environment construction in medical colleges and universities, software construction should be strengthened on the basis of existing public sharing experimental platform. According to the needs of students and teachers, the resource bank reflects the spirit of people-oriented, is conducive to stimulate students' enthusiasm for learning, mobilize teachers' enthusiasm for teaching, and thus improve the teaching effect. It is beneficial to curriculum reform and teaching mode innovation to closely link the construction of resource bank with the construction of excellent courses.

\section{Application Situations, Main Problems and Future Prospects of Digital Pathology Teaching Resources}

\subsection{Application Situations}

Under the background of global informatization, China has made great achievements in the construction of digital education resources, especially in Colleges and universities. Digital teaching has made great progress in the field of medical teaching and brought many benefits to it. In the process of digitized teaching of pathology morphology, the traditional morphology teaching process is replaced by digitized teaching, and even the pathological morphology teaching is completely separated from microscopy and general specimens. Therefore, a correct understanding of the role of digital pathology teaching, as well as how to use digital teaching in the teaching process, should be in-depth discussion. At present, many universities at home and abroad apply digitalization to the cumulative assessment of teaching and examination. In addition, through digital teaching, pre-class teachers can prepare rich image data and courseware. In the classroom, teachers can make use of the rich software function to make the course teaching more intuitive and more vivid. After class, through the intelligent homework system, students can more accurately analyze and grasp the situation of their mastery of knowledge, in addition to enhancing students' autonomous learning time, giving full play to students' subjective initiative. At present, virtual slices have been widely accepted as a teaching aids in many universities at home and abroad. Its application can solve the problems existing in conventional microscope teaching. For example, the number of slices is insufficient due to the loss of some typical slices and the inability to replenish the slices. The structure of the slices is inconsistent due to the depth of the slices in the same teaching wax block. Generally speaking, students and teachers are very willing to accept virtual slices as teaching tools, and it is very helpful to teaching. The virtual section was introduced into the teaching of Pathomorphological in various medical colleges, and good teaching effect was achieved by making good use of the teaching resources.

\subsection{Main Problems}

Pathological changes of diseases have diversity. Although digital teaching of pathological morphology has great advantages, many problems have been found in the process of practical teaching. Digital teaching system needs to be further improved at present, digital slices are widely used in medical colleges and universities in China. However, due to some limitations, the pixels of images are often not high, not clear enough, some of them appear color distortion, which affects the teaching effect. Therefore, in the process of using, we must constantly strengthen the improvement of the hardware system, constantly upgrade the software, so that the system is more stable. In addition, the software of pathological morphology digital teaching is not rich enough at present. We should develop the teaching software with the characteristics of the disease, image management software and some other personalized and meet special requirements of the software. To meet the needs of various aspects of teaching. Digital teaching will cause students to have insufficient understanding of pathological changes. In addition, it is easy for students to concentrate on problems other than 
learning when using computers connected to the Internet. Students' attention is hard to concentrate for a long time. Because of the abundant digital resources, students are more likely to be interested in browsing general specimens and slices of documents, and lose interest in the content of this lesson.

\subsection{Future Prospects}

Medical science is a very practical subject. Simple digital teaching cannot provide students with the understanding of pathological changes of diseases. It is very disadvantageous for students to observe only the images on the computer screen, but neglect the observation of the whole specimen and the section under the microscope. Despite the gradual improvement of technology, the resolution of digital images is far less than the actual observation. Many tissue structures and cell structures, such as intercellular bridges, lipofuscin, hemosiderin, etc., can be clearly observed only under a microscope, while it is not easy to observe on a computer screen and is easy to be ignored. Similarly, in the observation of general specimens, digital images tend to overlook some details. At the same time, due to reflection and refraction interference, some structures are prone to deformation, influence observation, or cause misunderstanding. Digital teaching is a new morphological teaching method, but digital teaching should be an important auxiliary means of pathological morphology teaching. In actual path morphology teaching, general specimens and tissue sections should still be the main teaching method. Teachers should explain the basic pathological changes of the disease according to the characteristics of each gross specimen and section, and mobilize students 'enthusiasm and classroom atmosphere by affecting each other between teachers and students. In this process, the flexible application of digital teaching means, as an auxiliary means in the teaching process, so that students can be observed through pictures, improve learning efficiency, and enhance the relationship between knowledge. The application of digital pathology technology can raise the level of medical diagnosis in China as a whole, and even alleviate the relationship between doctors and patients to a greater extent. We bring the digital pathological film technology into the construction of digital hospital, which can divide the digitization of pathological film into the important part of electronic medical record.

\section{Conclusion}

Digital teaching can combine many teaching links organically, greatly improve the quality of pathological morphology teaching, and stimulate the enthusiasm of students. Reasonable application of digital teaching means can effectively improve the effect of pathomorphological teaching. In the digital age, pathology teaching has new characteristics. Teachers must constantly learn new knowledge and ideas related to information technology to adapt to the rapid development of modern science and technology.

\section{Acknowledgement}

The paper is the results of "Planning Subject of the Education Department of Heilongjiang Province" named "Applications Research of Digital Pathology Teaching Resources under the Background of internet plus” (Grant No. GBC1317225).

\section{References}

[1] Zhang Jun, Wei Yuanyi, Yu Xiao, et al. Assessment of digital teaching applied in pathology experiment [J]. Laboratory Science, 2016, 19(3): 59-60.

[2] Lv Fudong, Kong Heli. Advantages and application in pathology teaching of digital slides [J]. China Medicine And Pharmacy, 2013, 3(23): 160-161+185.

[3] Ye Jing, Yuan Yuan, Zhao Yuanlin, et al. Digital teaching of pathological morphology: advantages and disadvantages [J]. Chinese Journal of Neurosurgical Disease Research, 2016, 15(1): 66-68. 
[4] Deng Yang, Bao Ji. The research prospect in computer- aided diagnosis of digital pathology [J]. Practical Journal of Clinical Medicine, 2017, 14(5): 10-12. 